\title{
Contamination du lait par Yersinia enterocolitica en Alsace
}

\author{
par \\ C. L. DELMAS* et D. J.M. VIDON**
}

\section{R é s u m é}

Une étude de la contamination du lait cru par Yersinia enterocolitica (YE) en Alsace a été réalisée d'une part sur des laits en vrac prélevés, soit dans les citernes des camions collecteurs, soit dans les bidons individuels des producteurs, et d'autre part sur des laits conditionnés prélevés dans le commerce. Cette enquête, menée entre février 1980 et juin 1981, et portant sur 233 échantillons, a montré que $77,2 \%$ des mélanges en camion citerne, $22,8 \%$ des laits à la production et $70 \%$ des laits conditionnés du commerce, soit en moyenne 54,5\%, sont contaminés par cette bactérie. En outre, l'analyse de 37 laits pasteurisés conditionnés nous a permis d'isoler YE de 3 échantillons.

Ce résultat a été obtenu notamment par une méthode combinant un pré-enrichissement prolongé à $4^{\circ} \mathrm{C}$ suivi d'un enrichissement en milieu tamponné à $\mathrm{pH}=8,3$ additionné d'azide de sodium et d'ampicilline, permettant de détecter un minimum de $15 \times 10^{2} \mathrm{YE}$ dans $100 \mathrm{ml}$ de lait cru. Une variation saisonnière de la contamination a été observée, avec un maximum en mars-avril, et un minimum en août.

Le typage de 158 souches a révélé que 97,3\% sont du chimiotype 1 , dont une proportion élevée $(23,2 \%)$ fermentent le lactose, et que les sérotypes prédominants sont 0:5 (33\%), 0:7 (24\%) et $0: 6(22,3 \%)$. Certains laits peuvent contenir jusqu'à 3 souches différentes, et une souche pigmentée en jaune a été isolée. La recherche

* Direction de la Consommation et de la Répression des Fraudes et Larebron, Chemin du Routoir - 67400 Illkirch Graffenstaden.

** Faculté de Pharmacie, Laboratoire de Bactériologie, B.P. 10 - 67048 Strasbourg cedex (France). 
de la virulence des souches par des épreuves in vitro de dépendance en calcium et d'auto-agglutination a été négative.

Mots clés

Yersinia enterocolitica - Lait cru - Lait pasteurisé - Virulence.

Titre abrégé

Yersinia enterocolitica dans le lait.

\section{S u m m a r y}

\section{OCCURENCE OF YERSINIA ENTEROCOLITICA IN MILK \\ OF ALSACE}

A study of the milk contamination by Y. enterocolitica (YE) in Alsace was carried out, either in bulk raw milk sampled from tanks on their arrival at dairy plant, and from producer individual cans, or in processed raw or pasteurized milk picked up in retailers. This investigation, led from february 1980 through june 1981 on 233 samples of raw milk, showed that $77.2 \%$ of mixed milk from tanks, $22.8 \%$ of milk from individual cans, and $70 \%$ of processed raw milk, i.e. an average of $54.5 \%$, contained this bacteria. Moreover, 3 of 37 pasteurized milks were contamined by YE. These results were obtained especially by a method combinating a pre-enrichment at $4^{\circ} \mathrm{C}$ followed by an enrichment in a $p H=8.3$ buffered-broth added with sodium azide and ampicillin $(5 \mu \mathrm{g} / \mathrm{ml})$, allowing to detect about $15.10^{2}$ c.f.u. of $Y E$ in $100 \mathrm{ml}$ of raw milk. A seasonal variation of the contamination rate was observed, with a maximum in marchapril, and a minimum in august. The typing of 158 isolates revealed that $97.3 \%$ belonged to biotype 1, of which a high percentage fermented lactose $(23,2 \%)$, and that the predominating serotypes were $0: 5(33 \%), 0: 7,8(24 \%)$ and $0: 6(22.3 \%)$. Some milk samples could contain up to 3 different strains, and a yellow-pigmented strain was isolated. Study of the virulence of the strains by "in vitro » tests of calcium dependance and auto-agglutination was negative.

Key words

Yersinia enterocolitica - Raw milk - Pasteurized milk - Virulence.

\section{INTRODUCTION}

La connaissance de Yersinia enterocolitica $(Y E)$ remonte à une quarantaine d'années environ, mais pendant longtemps son identité est demeurée imprécise, jusqu'à ce que Frédériksen propose, en 1964, sa dénomination admise à l'heure actuelle. Le rôle pathogène de cette entérobactérie revêt les aspects les plus divers, mais la forme 
prédominante de l'infection reste l'entérocolite du jeune enfant. Les enquêtes épidémiologiques, en particulier celles de Mollaret en France, ont montré l'importance des rongeurs sauvages, du sol et de l'eau comme réservoirs de germes (Mollaret, 1976). Cependant, il faut distinguer les souches non adaptées de l'environnement, au faible pouvoir pathogène, de celles adaptées à l'homme, dont les sérotypes sont différents des premières, essentiellement 0:3 et 0:9 en Europe, et responsables de manifestations digestives et même d'infections généralisées septicémiques lorsque le terrain est immunodéprimé. La contamination fréquente du porc ne serait, d'après Mollaret, qu'un reflet, et non une cause, de la contamination humaine.

Bien que le premier isolement de YE du lait semble remonter à 1972 (Sarrouy, 1972), ce n'est qu'à partir de 1975 que des recherches systématiques commencent à être entreprises dans différents pays tels que la Tchécoslovaquie (Aldova et al., 1975), le Canada (Schiemann et Toma, 1978), l'Australie (Hughes, 1979; Hughes et Jensen, 1981), la France (Vidon et Delmas, 1981 ; Lauret, 1981) et le Danemark (Christensen, 1982). Ces enquêtes concernent le lait cru, mais la présence de YE dans le lait pasteurisé a également été rapportée par quelques auteurs (Hughes, 1980; Schiemann, 1978; Delmas, 1982).

Nous rapportons ici les résultats de recherches effectuées dans le lait cru en Alsace entre les mois de février 1980 et juin 1981, qui ont fait l'objet d'une thèse de Doctorat soutenue par l'un de nous (Delmas, 1982), et dont une partie a déjà été publiée (Vidon et Delmas, 1981).

\section{MATERIEL ET METHODES}

\section{Echantillons de lait}

Trois types d'échantillons de lait cru ont été prélevés pour analyse :

- lait en vrac dans une laiterie centrale;

- lait en vrac chez les producteurs;

- lait conditionné chez les détaillants.

a) LAIT EN VRAC DANS UNE LAITERIE CENTRALE

Le lait est recueilli le matin dans les compartiments de camions citernes réfrigérés ayant effectué leur collecte dans les environs de Strasbourg. Il s'agit donc de mélanges correspondant à la production de 20 à 50 éleveurs récoltée le jour même. Le volume de l'échantillon recueilli dans un flacon stérile est de $250 \mathrm{ml}$.

b) LAIT EN VRAC CHEZ LES PRODUCTEURS

Le lait est prélevé dans une coopérative laitière regroupant 25 producteurs, dans une petite agglomération du nord de l'Alsace, 
que nous désignerons par "village $\mathrm{X}$ ". Les prélèvements sont effectués dans les bidons individuels des producteurs à leur arrivée à la coopérative. Il s'agit là encore de la traite du matin, pratiquée moins d'une heure auparavant, dont le volume prélevé est aussi de $250 \mathrm{ml}$.

c) LAIT CONDItionné

Le lait cru conditionné en "tétrabrik " ou en sac de matière plastique thermosoudé est prélevé dans le commerce par les inspecteurs de la Direction de la Consommation et de la Répression des fraudes. Au laboratoire, les récipients sont ouverts aseptiquement, et $125 \mathrm{ml}$ de lait sont recueillis dans une éprouvette stérile.

De plus, des échantillons de lait pasteurisé conditionné en cartons d'un litre provenant de la laiterie centrale sont analysés. Chaque échantillon est représentatif de la production d'une journée. Le carton est ouvert avec précautions, et le lait $(125 \mathrm{ml})$ recueilli aseptiquement comme ci-dessus. La réaction de la phosphatase est vérifiée systématiquement.

\section{Enrichissement, isolement et identification des Yersinia}

a) Milieux D'enrichissement

Trois milieux d'enrichissement ont été utilisés :

- Eau peptonée tamponnée à concentration double (EPT) :

Pastone (Institut Pasteur de Lille, ou IPL) $20 \mathrm{~g}$

$\mathrm{NaCl}$

$\mathrm{Na}_{2} \mathrm{H} \quad \mathrm{PO}_{4}$

$\mathrm{NaH}_{2} \mathrm{PO}_{4}$

eau distillée

$\mathrm{pH}$ ajusté à 7,5

Stérilisation à $120^{\circ} \mathrm{C}$ pendant $20 \mathrm{~min}$.

- Milieu de Rappaport modifié (RMB) :

Solution $\mathrm{A}$ : eau peptonée à $1 \%$ (Pastone IPL).

- $\quad$ B : $\mathrm{Na}_{2} \mathrm{H} \mathrm{PO}_{4} \mathrm{M} / 15$.

- $\quad$ C: $\mathrm{MgCl}_{2}, 6 \mathrm{H}_{2} \mathrm{O}$ à $40 \%$.

- $\quad \mathrm{D}$ : vert malachite à $0,2 \%$.

Stériliser séparément les solutions $\mathrm{A}, \mathrm{B}, \mathrm{C}$ et $\mathrm{D}$ à $115^{\circ} \mathrm{C}$ pendant $20 \mathrm{~min}$.

Milieu final : $155 \mathrm{ml} \mathrm{A}+40 \mathrm{ml} \mathrm{B}+53 \mathrm{ml} \mathrm{C}+1,6 \mathrm{ml} \mathrm{D}$. $\mathrm{pH}$ final : 6,5.

- Milieu à l'azide-ampicilline (PSTA)

Base (PST) : Pastone (IPL)

Tris (hydroxy-méthylaminométhane)

Saccharose

eau distillée 
Stériliser à $115^{\circ} \mathrm{C}$ pendant $20 \mathrm{~min}$.

Milieu complet (PSTA) :

ajouter extemporanément à 11 de PST les solutions aqueuses :

— azide de sodium à $1 \%$ stérilisée par filtration $20 \mathrm{ml}$

— ampicilline à $0,05 \%$ stérilisée par filtration $10 \mathrm{ml}$

— vert brillant à $0,125 \%$. Stériliser à $115^{\circ}$ pendant $20 \mathrm{~min}$

$10 \mathrm{ml}$

$\mathrm{pH}$ final : $8,4 \pm 0,1$.

\section{b) PRÉ-ENRICHISSEMENT}

$125 \mathrm{ml}$ de lait sont ajoutés à $125 \mathrm{ml}$ d'EPT. Le mélange est placé à la chambre froide à $4^{\circ} \mathrm{C}$ pendant une durée maximum de 4 semaines.

c) ENRICHISSEMENT

Après 7, 14, 21 et 28 jours de pré-enrichissement à $4^{\circ} \mathrm{C}$, on prélève $1 \mathrm{ml}$ de mélange pré-enrichi qu'on inocule :

- soit dans $20 \mathrm{ml}$ de RMB (méthode $\mathrm{R}$ );

- soit dans $20 \mathrm{ml}$ de PSTA (méthode A).

Le milieu RMB est maintenu pendant 3 jours à température du laboratoire.

Le milieu PSTA est placé en incubation à $28^{\circ} \mathrm{C}$ pendant $48 \mathrm{~h}$.

d) ISOLEMENT

Une anse prélevée dans le milieu d'enrichissement est épuisée en strie sur géloses Hektoen (IPL) additionnée de $5 \mu \mathrm{g} / \mathrm{ml}$ d'ampicilline.

En outre, une anse prélevée dans le mélange de pré-enrichissement est épuisée de la même manière sur un milieu d'isolement identique tous les 7 jours durant la phase de pré-enrichissement (méthode D).

e) IdENTIFICATION

5 à 10 colonies de couleur jaune-orange apparues sur milieu Hektoen après $48 \mathrm{~h}$ d'incubation à $28^{\circ} \mathrm{C}$ sont étudiées individuellement. La mise en évidence des caractères suivants permet de faire un premier criblage :

$$
\begin{gathered}
\text { uréase }+ \\
\text { tryptophane désaminase - } \\
\text { ONPG + } \\
\text { lysine décarboxylase - }
\end{gathered}
$$

L'identification des colonies suspectes est complétée à l'aide des plaques API 20E pour Enterobacteriaceae, et par l'étude de la mobilité différentielle à $22^{\circ} \mathrm{C}$ et à $37^{\circ} \mathrm{C}$. En outre, on recherche la fermentation éventuelle du lactose (sur milieu de Hugh et Leifson) et du 
raffinose, l'utilisation du citrate de Simmons, et la production de lécithinase (sur milieu à l'œuf) à $28^{\circ} \mathrm{C}$ pendant $48 \mathrm{~h}$.

Les souches ainsi identifiées sont enfin envoyées au Centre National des Yersinia (Institut Pasteur, $\operatorname{Pr}$ H. H. Mollaret) pour typage complet.

La sensibilité des souches aux antibiotiques et au mercure est également examinée par antibiogramme sur gélose Mueller-Hinton.

\section{RESULTATS}

Entre le mois de février 1980 et le mois de juin 1981, 233 échantillons de lait cru ont été analysés, dont 101 prélevés dans la laiterie centrale, 92 chez les producteurs du village $X$, et 40 chez les détaillants du commerce (lait conditionné). YE a été isolée de 127 de ces échantillons, ce qui représente une contamination moyenne de $54,5 \%$. Cependant, le pourcentage de laits contaminés varie selon le type d'échantillon, ceux prélevés dans les bidons des producteurs du village $X$ apparaissant les moins fréquemment infectés $(22,8 \%)$, et ceux recueillis à la laiterie centrale montrant le taux de contamination le plus élevé $(77,2 \%)$.

D'autre part, sur 37 laits pasteurisés conditionnés de productions différentes, 3 étaient contaminés par $\mathrm{YE}$, soit $8,1 \%$.

Les résultats varient en fonction de la période de l'année, avec un maximum de positivité se situant en mars-avril (48\% de positifs chez les producteurs, $92,3 \%$ à la laiterie centrale, et $100 \%$ des laits conditionnés du commerce), et un minimum en août $(4,55 \%$ chez les producteurs du village $\mathrm{X}$ ) (tab. 1).

Enfin, la positivité des résultats dépend en grande partie de la méthode d'enrichissement utilisée. La méthode A donne régulièrement les résultats les plus élevés, et la méthode $\mathrm{R}$ les résultats les plus bas. Le détail des résultats obtenus, échantillon par échantillon, montre que la méthode A n'est en défaut que dans 7 cas seulement, alors que la méthode D est défaillante dans 60 cas, et la méthode $\mathrm{R}$ dans 79 cas (tab. 2).

\section{Sensibilité des méthodes d'analyse}

Afin d'évaluer le seuil de sensibilité des méthodes utilisées, nous avons contaminé artificiellement un lait cru avec différents inoculums, de manière à obtenir des concentrations finales respectives de $15 \times 10^{4}, 15 \times 10^{3}, 15 \times 10^{2}, 15 \times 10^{1}$ et 15 germes (ou unités formant colonie) pour $100 \mathrm{ml}$. La souche de YE utilisée pour ces expériences a été sélectionnée pour son chimiotype rare (chimiotype 3) afin d'éviter toute interférence avec une autre souche susceptible de contaminer le lait cru choisi. 


\section{TABLEAU 1 - TABLE 1}

Isolement de $Y$. enterocolitica dans le lait cru.

Résultats des analyses effectuées par 3 méthodes entre février 1980 et juin 1981, sur des échantillons prélevés en camions-citernes (laiterie), en bidons individuels du village $\mathrm{X}$ (producteurs) ou en récipients conditionnés (commerce).

Isolation of Y. enterocolitica in raw milk. Results of analysis carried out by 3 procedures from february 1980 through june 1981, in milk sampled from tanks (central dairy), individual cans of the $X$ village (producers) or processed (retailers).

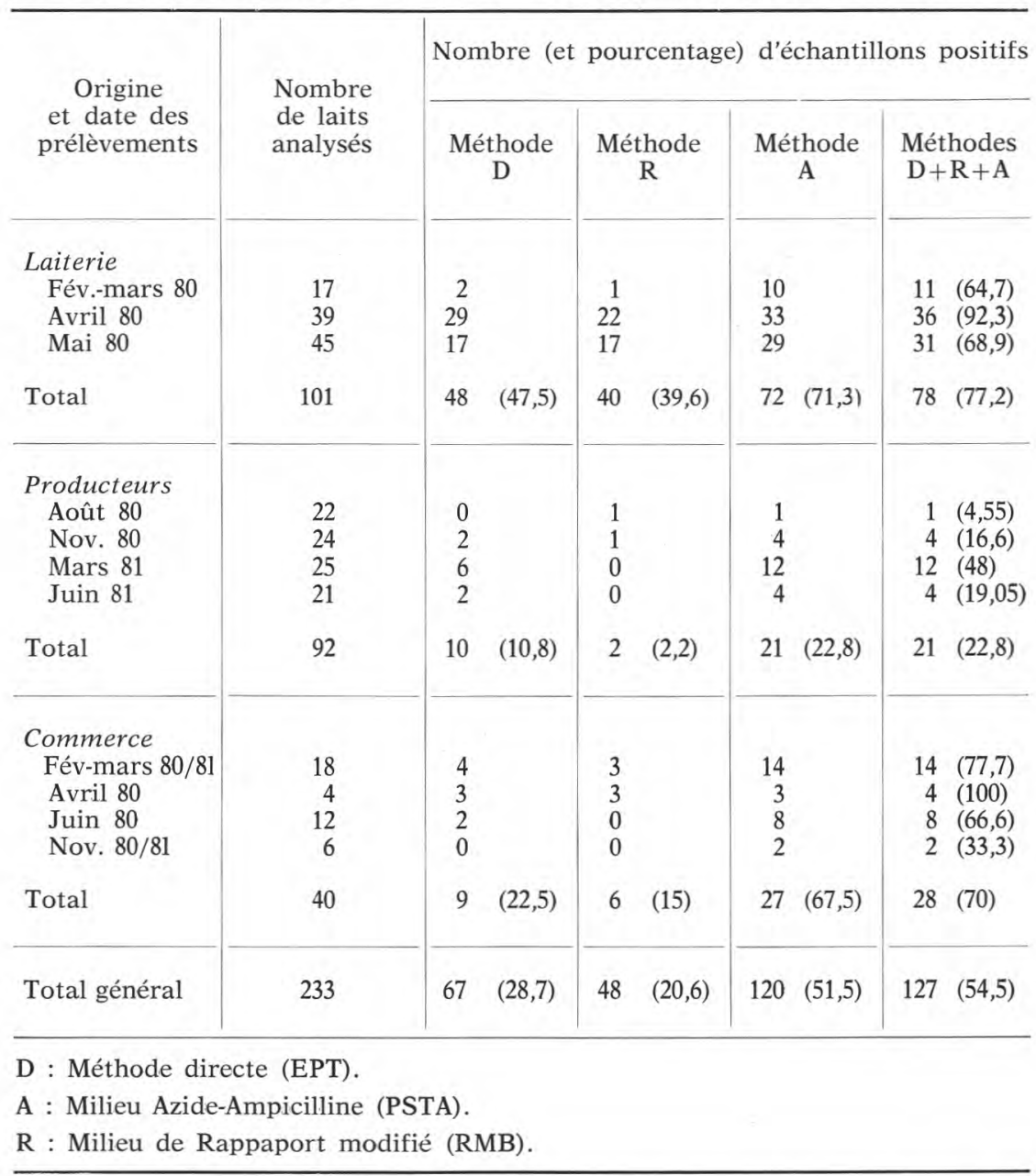

Le lait ainsi ensemencé, a été analysé par les différentes méthodes décrites précédemment, immédiatement et après conservation en chambre froide à $4^{\circ} \mathrm{C}$ pendant $7,14,21$ et $31 \mathrm{j}$. 
TABLEAU 2 - TABLE 2

Influence de la méthode d'analyse sur la fréquence d'isolement de $Y$. enterocolitica du lait cru.

Influence of the procedure of analysis on the frequency of isolation of Y. enterocolitica from raw milk.

\begin{tabular}{|c|c|c|c|}
\hline $\begin{array}{l}\text { Nombre d'échantillons } \\
\text { positifs }\end{array}$ & $\begin{array}{l}\text { Méthode } \\
\text { D }\end{array}$ & $\begin{array}{l}\text { Méthode } \\
\quad \mathrm{R}\end{array}$ & $\begin{array}{l}\text { Méthode } \\
\text { A }\end{array}$ \\
\hline $\begin{array}{r}48 \\
36 \\
26 \\
10 \\
5 \\
2 \\
0\end{array}$ & $\begin{array}{l}\overline{+} \\
+ \\
- \\
+ \\
+ \\
+\end{array}$ & $\begin{array}{l}-\bar{t} \\
\frac{+}{+} \\
\frac{+}{+} \\
+\end{array}$ & $\begin{array}{l}+ \\
+ \\
+ \\
+ \\
- \\
- \\
-\end{array}$ \\
\hline
\end{tabular}

Nous avons simultanément évalué l'efficacité de l'ampicilline en rajoutant cet antibiotique dans le milieu RMB à la même concentration que dans le milieu PSTA, et en le supprimant du milieu PSTA et de la gélose Hektoen.

Les résultats montrent (tab. 3) que la méthode A avec ampicilline dans le milieu PSTA est la plus sensible. En effet, il est possible d'isoler YE dans le lait après $14 \mathrm{j}$ d'enrichissement avec inoculum initial de $15 \times 10^{2}$ germes pour $100 \mathrm{ml}$. Les autres méthodes ont toutes été en défaut avec cet inoculum.

Avec un inoculum 10 fois supérieur, il est possible d'isoler YE plus précocément par la méthode $\mathrm{A}$, au $7^{\mathrm{e}} \mathrm{j}$ de conservation à froid, à condition de supprimer l'ampicilline du milieu PSTA.

\section{Chimiotypes, sérotypes, lysotypes}

Sur un total de 272 souches isolées des 127 laits crus positifs, 158 ont été typées au Centre National des Yersinia. Parmi celles-ci, 112 ont été retenues sur la base de l'un des critères suivants :

- provenance d'un échantillon de lait indépendant, pour les souches de caractères identiques;

- caractères biochimiques, sérotypes ou lysotypes différents, pour les souches isolées du même échantillon de lait.

A ces 112 souches de YE sensu-stricto, il faut ajouter les 3 souches isolées des laits pasteurisés, et 3 Yersinia enterocoliticalike appartenant aux nouvelles espèces $Y$. frederiksenii (2 souches) 


\section{TABLEAU 3 - TABLE 3}

Evaluation du seuil de sensibilité des méthodes d'analyse au cours de la recherche de $Y$. enterocolitica dans un lait cru ensemencé avec différents inoculums.

Evaluation of the sensitivity of the procedures during the search of Y. enterocolitica in a raw milk inoculated with different concentrations of bacteria.

\begin{tabular}{|c|c|c|c|c|c|c|c|}
\hline \multirow{2}{*}{\multicolumn{2}{|c|}{$\begin{array}{l}\text { Inoculum } \\
\text { pour } 100 \mathrm{ml} \\
\text { de lait }\end{array}$}} & \multirow{2}{*}{$\begin{array}{l}\text { Milieux } \\
\text { d'isolement }\end{array}$} & \multicolumn{5}{|c|}{$\begin{array}{l}\text { Durée de l'enrichissement (en jours) } \\
\text { conduisant à l'isolement de YE }\end{array}$} \\
\hline & & & $\mathrm{D}$ & A & $\mathrm{R}$ & AS & RA \\
\hline \multirow{2}{*}{\multicolumn{2}{|c|}{$\begin{array}{c}0 \\
\text { (témoin) }\end{array}$}} & $\mathrm{H}$ & - & - & - & - & - \\
\hline & & HA & - & - & - & - & - \\
\hline \multirow{2}{*}{\multicolumn{2}{|c|}{15}} & $\mathrm{H}$ & - & - & - & - & - \\
\hline & & HA & - & - & - & - & - \\
\hline \multirow{2}{*}{\multicolumn{2}{|c|}{$15 \times 10^{1}$}} & $\mathrm{H}$ & - & - & - & - & - \\
\hline & & HA & - & - & - & - & - \\
\hline \multirow{2}{*}{\multicolumn{2}{|c|}{$15 \times 10^{2}$}} & $\mathrm{H}$ & - & 14 & - & - & - \\
\hline & & HA & - & 14 & - & - & - \\
\hline \multirow{2}{*}{\multicolumn{2}{|c|}{$15 \times 10^{3}$}} & $\mathrm{H}$ & - & 14 & - & - & - \\
\hline & & HA & - & 14 & - & $7-14-21$ & - \\
\hline \multirow{2}{*}{\multicolumn{2}{|c|}{$15 \times 10^{4}$}} & $\mathrm{H}$ & - & 14 & - & 7 & - \\
\hline & & HA & 14 & $7-14-21$ & - & $7-14-21$ & 14 \\
\hline \multicolumn{8}{|c|}{ D : méthode directe (EPT). } \\
\hline \multicolumn{8}{|c|}{ u à l'Azide-A } \\
\hline \multicolumn{8}{|c|}{ : milieu de Rappaport modifié (RMB). } \\
\hline \multicolumn{8}{|c|}{ milieu à l'Azide sans ampicilline. } \\
\hline \multicolumn{8}{|c|}{ : milieu de Rappaport modifié + ampicilliı } \\
\hline \multicolumn{8}{|c|}{ H : gélose Hektoen. } \\
\hline \multicolumn{8}{|c|}{ HA : gélose Hektoen + ampicilline. } \\
\hline \multicolumn{8}{|c|}{ - $\quad:$ recherche négative. } \\
\hline \multicolumn{8}{|c|}{ 7-14-21 : recherche positive après $\mathrm{x}$ jours d'enrichissement à $4^{\circ} \mathrm{C}$. } \\
\hline
\end{tabular}


et Y. kristensenii (1 souche) (Ursing et al., 1980; Bercovier et al., 1980).

\section{Chimiotypes}

Les caractères biochimiques de ces souches sont résumés sur le tableau 4, duquel sont exclues les 3 souches YE-like fermentant le rhamnose et les 3 souches issues des laits pasteurisés.

Le trait à souligner dans ce tableau est la fréquence singulièrement élevée des souches fermentant le lactose $(23,2 \%)$ étroitement corrélée à la fermentation du raffinose $(22,3 \%)$; une seule souche fermentant le lactose, mais non le raffinose, échappe à la règle.

\section{TABLEAU 4 - TABLE 4}

Principaux caractères biochimiques des souches de $Y$. enterocolitica sensu-stricto isolées du lait cru.

Relevant biochemical characters of the strains of $\mathrm{Y}$. enterocolitica isolated from raw milk.

\begin{tabular}{|c|c|c|}
\hline Caractères biochimiques & Positifs & $\%$ \\
\hline $\begin{array}{l}\text { Lécithinase } \\
\text { Uréase } \\
\text { Tryptophane désaminase (TDA) } \\
\text { ONPG } \\
\text { Mobilité à } 22^{\circ} \mathrm{C} \\
\text { Mobilité à } 37^{\circ} \mathrm{C} \\
\text { Voges-Proskauer (acétoïne à } 22 \text { et } 28^{\circ} \mathrm{C} \text { ) } \\
\text { Citrate Simmons } \\
\text { Indole } \\
\text { Lysine décarboxylase (LDC) } \\
\text { Ornithine décarboxylase (ODC) } \\
\text { Arginine dihydrolase (ADH) } \\
\text { Lactose oxydationa } \\
\text { Lactose fermentationa } \\
\mathrm{H}_{2} \mathrm{~S} \\
\text { Gélatinase } \\
\text { Glucose } \\
\text { Mannitol } \\
\text { Inositol } \\
\text { Sorbitol } \\
\text { Rhamnose } \\
\text { Saccharose } \\
\text { Mélibiose } \\
\text { Amygdaline } \\
\text { Arabinose } \\
\text { Raffinose }\end{array}$ & $\begin{array}{r}109 \\
112 \\
0 \\
112 \\
110 \\
0 \\
106 \\
29 \\
109 \\
0 \\
112 \\
0 \\
86 \\
26 \\
0 \\
0 \\
0 \\
112 \\
112 \\
107 \\
109 \\
0 \\
112 \\
0 \\
112 \\
112 \\
25\end{array}$ & $\begin{array}{c}97,3 \\
100 \\
0 \\
100 \\
98,2 \\
0 \\
94,6 \\
25,9 \\
97,3 \\
0 \\
100 \\
0 \\
76,8 \\
23,2 \\
0 \\
0 \\
100 \\
100 \\
95,5 \\
97,3 \\
0 \\
100 \\
0 \\
100 \\
100 \\
22,3\end{array}$ \\
\hline
\end{tabular}

N.B. : Les caractères biochimiques sont étudiés à $28 \pm 1^{\circ} \mathrm{C}$ exceptés la mobilité et Voges -Proskauer.

a : Milieu Oxydation/Fermentation de Hugh et Leifson. 
L'indole est produite par 109 souches (97,3\%) lesquelles sont également lécithinase positive, ces deux caractères permettant de les classer dans le chimiotype 1 (Wauters, 1970). Les 3 autres souches indole et lécithinase négatives appartiennent au chimiotype 3. Notons que ces 3 souches ont été isolées de laits provenant du village $\mathrm{X}$, et qu'aucune des 32 souches de cette même provenance ne fermente le lactose.

\section{Sérotypes}

Les 112 souches peuvent être groupées en 8 sérotypes, auxquels il convient d'ajouter 4 souches non agglutinables, donc de sérotype non identifié, et 2 souches rugueuses non typables. Les sérotypes qui prédominent sont : 0:5 (33\%), 0:7,8 (24\%) et 0:6 (22,3\%), les autres sérotypes étant beaucoup plus rares et n'atteignant pas $6 \%$ (tab. 5).

\section{Lysotypes}

Les 112 souches se répartissent inégalement entre les lysotypes $\mathrm{Xz}(76,8 \%)$ et $\mathrm{Xo}(23,2 \%)$ (tab. 5).

\section{TABLEAU 5 -TABLE 5}

Chimiotypes, sérotypes et lysotypes de 112 souches de $Y$. enterocolitica isolées du lait cru.

Biotypes, serotypes and bacteriophage types of 112 strains of $\mathrm{Y}$. enterocolitica isolated from raw milk.

\begin{tabular}{|c|c|c|}
\hline Types & Nombre & $\%$ \\
\hline $\begin{array}{c}\text { Chimiotypes } \\
1 \\
3\end{array}$ & $\begin{array}{r}109 \\
3\end{array}$ & $\begin{array}{r}97,3 \\
2,7\end{array}$ \\
\hline $\begin{array}{l}\text { Sérotypes } \\
\quad 4,32 \\
5 \\
5,27 \\
6 \\
6,30 \\
7,8 \\
13,7,8,19 \\
27 \\
\quad \text { NAG } \\
\text { R }\end{array}$ & $\begin{array}{r}3 \\
37 \\
3 \\
25 \\
3 \\
27 \\
2 \\
2 \\
6 \\
4 \\
2\end{array}$ & $\begin{array}{c}2,7 \\
33 \\
2,7 \\
22,3 \\
2,7 \\
24 \\
1,8 \\
5,4 \\
3,6 \\
1,8\end{array}$ \\
\hline $\begin{array}{c}\text { Lysotypes } \\
\mathrm{X}_{\mathrm{Z}} \\
\mathrm{X}_{0}\end{array}$ & $\begin{array}{l}86 \\
26\end{array}$ & $\begin{array}{l}76,8 \\
23,2\end{array}$ \\
\hline $\begin{array}{l}\text { NAG : non agglut } \\
\mathrm{R}: \text { rugueuse. }\end{array}$ & & \\
\hline
\end{tabular}




\section{Sensibilité aux antibiotiques et au mercure}

L'étude de la sensibilité in vitro aux antibiotiques de 170 souches a montré que toutes les souches sont résistantes à l'ampicilline, mais sensibles à la streptomycine, au chloramphénicol, à la tétracycline et aux sulfamides. Seulement $6 \%$ des souches se sont montrées sensibles à la carbénicilline, parmi lesquelles la moitié sont du rare chimiotype 3 .

Toutes les souches sont sensibles au chlorure mercurique, à l'exception d'une seule (YE 138 A14) qui est résistante à la fois au chlorure mercurique et au mercurochrome, mais sensible au borate de phényl mercure et au merthiolate de sodium (Vidon, Lett et Delmas, 1981). Cette souche fait actuellement l'objet d'une étude génétique dans notre laboratoire.

\section{Pigmentation}

Classiquement, les colonies $\mathrm{S}$ de $\mathrm{YE}$ sur gélose nutritive présentent l'aspect typique de colonies d'entérobactéries, rondes, lisses, brillantes, translucides à légèrement opaques, non pigmentées. L'une

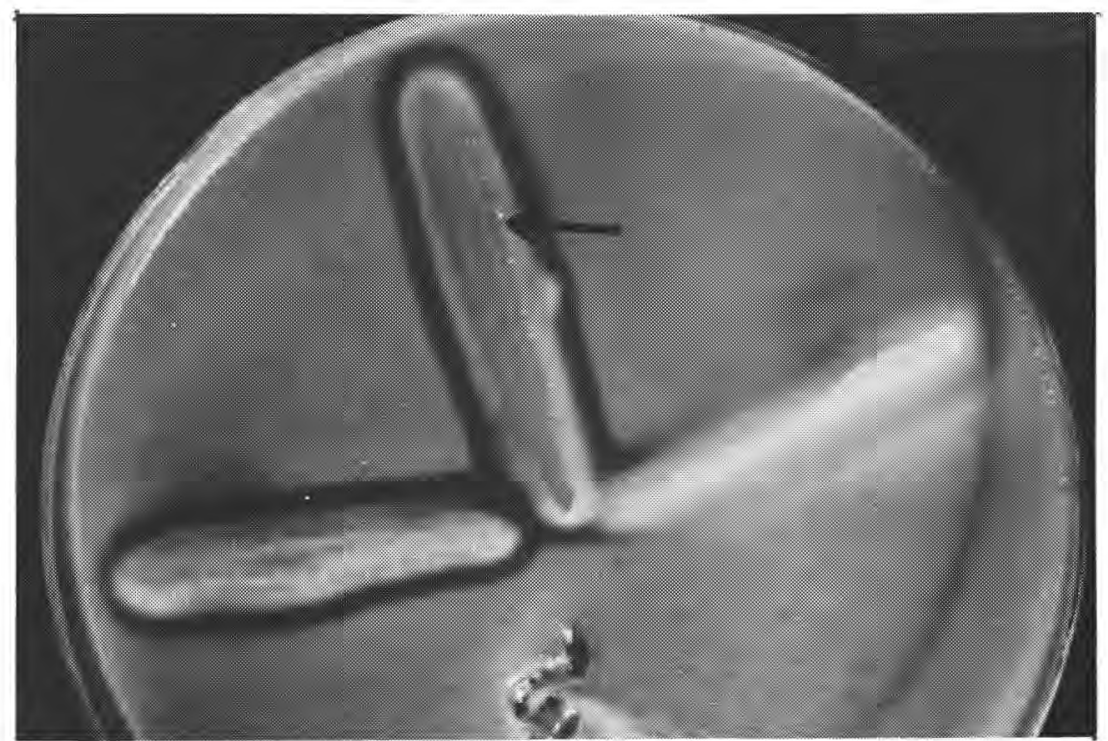

Fig. 1

Production d'un pigment jaune par une souche de $Y$. enterocolitica (YE 195 A14 J) isolée du lait cru (culture sur gélose à l'œuf).

Yellow pigment produced by a strain of Y. enterocolitica (YE 195 A14 J) isolated from raw milk (egg yolk agar). 
des souches que nous avons isolées du lait (YE 195 A14J) produit un pigment endocellulaire jaune. Cette souche était d'ailleurs associée à une autre souche non pigmentée typique. La pigmentation de YE 195 A14J est accentuée sur gélose au sang, gélose à l'œuf, et milieu à la pomme de terre.

\section{DISCUSSION ET CONCLUSION}

Notre enquête montre pour la première fois le taux élevé de contamination du lait cru par YE dans l'est de la France. En effet, une moyenne de $54,8 \%$ des 233 échantillons prélevés soit en laiterie centrale, soit chez les détaillants, soit à la source de la production dans un petit village du Bas-Rhin, se sont révélés positifs à la recherche de cette bactérie. Nous avons antérieurement publié des résultats d'une partie de cette enquête, portant sur un nombre limité d'échantillons (71 laits) prélevés sur une courte période de l'année 1980 (février-avril) (Vidon et Delmas, 1981). Le pourcentage d'échantillons de lait positifs atteignait $81,4 \%$, et même $92,3 \%$ si l'on considérait les résultats d'une série de 39 prélèvements effectués dans la seule journée du 28 avril 1980. Il est clair que le tassement relatif du taux de positivité sur l'ensemble de l'enquête, qui recouvre 17 mois (février 1980 à juin 1981), résulte de l'intégration d'analyses de laits prélevés durant la saison chaude. Or, il n'est pas surprenant, comme notre étude le montre, que la contamination du lait par une bactérie psychrotrophe comme YE subisse des variations saisonnières, avec un maximum pendant l'hiver et le printemps, et un minimum en été-automne.

Le typage d'une grande partie des souches a montré qu'aucune de celles-ci n'appartient aux sérotypes $0: 3$ et $0: 9$ classiquement responsables des infections humaines en Europe. Ce constat est indiscutablement rassurant, bien qu'il faille se garder de toute conclusion précipitée quant à l'innocuité complète des souches isolées. En effet, l'adaptation de YE est actuellement en cours d'évolution, et ce qui était admis hier dans le domaine du pouvoir pathogène de cette bactérie, comme de beaucoup d'autres d'ailleurs, ne sera pas nécessairement encore reconnu valable demain. Divers cas de diarrhées ou de gastroentérites reliés à l'isolement de souches de sérotype $0: 8$ ou $0: 5,27$ dans les selles des malades ont été rapportés dans la littérature (Gutman et al., 1973; Black et al., 1978; Degrace et al., 1976; Kasatiya, 1976) et récemment, une épidémie hospitalière de diarrhée associée au sérotype 0:5 a été signalée au Canada (Ratnam et al., 1982). En France, durant l'année 1980, Mégraud et al. ont isolé 8 souches de sérotypes "non adaptés ", dont 4 de type 0:5, à partir de selles d'enfants présentant une symptomatologie abdo- 
minale (Mégraud et al., 1981), et Mollaret (communication personnelle) estime que ce sérotype semble en cours d'émergence dans les infections humaines. Faut-il en conclure que la présence aussi fréquente de YE dans le lait, avec prédominance de souches 0:5, constitue un risque sérieux pour la santé publique? Il est utile de rappeler que la pathologie de ce germe est souvent liée à un terrain particulier : jeune âge, immunodépression, cirrhose, antigène d'histocompatibilité HLA B 27, etc. D'autre part, la virulence des souches entre également en ligne de compte, celle-ci semblant en partie sous le contrôle génétique d'un plasmide (Portnoy, Moseley et Falkow, 1981). Des tests " in vitro " simples ont été proposés pour déterminer la virulence des souches : auto-agglutinabilité à $37^{\circ} \mathrm{C}$ en milieu de culture de tissus (Laird et Cavanaugh, 1980), dépendance en calcium à $37^{\circ} \mathrm{C}$ (Gemski, Lazère et Casey, 1980). Nous avons nousmême effectué ces épreuves sur l'ensemble de nos souches, mais toutes ont été négatives, semblant attester l'absence de virulence (Delmas, 1982).

La réponse à la question posée ci-dessus doit donc être très nuancée. Il est certain que la consommation de lait cru par les enfants, ou les individus à haut risque (vieillards, cirrhotiques, sujets aux défenses immunitaires réduites ou porteurs de l'antigène B27) doit être rigoureusement prohibée. En revanche, nous ne disposons pour le moment d'aucun élément biologique indiscutable qui nous permette d'adopter une position claire en ce qui concerne le risque encouru vis-à-vis des Yersinioses par l'adulte sain consommant ce lait cru.

La pasteurisation apporte, à cet égard, un facteur de sécurité indéniable, mais elle n'élimine pas totalement la contamination par YE. En effet, un sondage réalisé sur 37 laits pasteurisés conditionnés nous a permis d'isoler cette bactérie de 3 d'entre eux.

Différents auteurs ont signalé ces dernières années la présence de YE dans le lait. Au Canada, Schieman et Toma ont retrouvé cette bactérie dans $31 \%$ des laits crus, et $0,4 \%$ des laits pasteurisés. En Australie, Hughes a isolé 16 souches à partir d'un nombre non précisé de laits crus, et a montré également la contamination du lait pasteurisé. Même au Danemark, où les produits laitiers jouissent d'une réputation prestigieuse, Christensen a trouvé $10 \%$ de laits contaminés à la production, ce pourcentage s'élevant à $16 \%$ lorsque les laits examinés sont des mélanges. En France enfin, l'enquête de Lauret effectuée pendant l'hiver 1980-1981 das le bassin parisien fait état de $8,7 \%$ de laits crus contaminés sur 103 échantillons analysés.

Il ressort donc de notre étude que la contamination du lait cru par YE en Alsace atteint un niveau élevé, rapporté nulle part ailleurs jusqu'ici, en France comme à l'étranger. Le long travail préliminaire 
consacré à l'amélioration de la technique d'isolement de cette bactérie n'est sans doute pas étranger à ce surprenant résultat. L'emploi du milieu Hektoen additionné d'ampicilline, quoique un peu plus sélectif que les milieux SS (Salmonella-Shigella) ou DCL (Desoxycholate-citrate-lactose), nous a permis de ne pas écarter les souches fermentant le lactose qui représentent le tiers de l'ensemble des souches isolées. D'aure part, le milieu d'enrichissement PSTA tamponné à $\mathrm{pH}=8,3$ par le Tris est probablement plus favorable que les autres milieux utilisés habituellement grâce à son $\mathrm{pH}$ plus élevé, du moins pour les souches laitières.

Soulignons enfin qu'un même lait peut être contaminé par plusieurs souches de YE différentes. Ainsi, à partir de 4 échantillons de lait totalement indépendants, nous avons isolé dans chacun d'entre eux 3 souches différant entre elles par le chimiotype, le sérotype ou le lysotype. De même, dans 10 échantillons de laits différents, nous avons isolé 2 souches non identiques.

Ces résultats soulèvent le problème de l'origine de la contamination du lait par YE. Parmi les diverses sources possibles, deux d'entre elles, fécale et hydrique, nous paraissent les plus probables. Pour étayer la première hypothèse, il faudrait montrer le portage fécal de YE par les bovidés, ce qui sortait des limites de notre enquête. Quant à l'origine hydrique, elle est tout à fait plausible compte tenu de l'existence de cette espèce bactérienne dans les eaux de surface (Mélis et Mollaret, 1981). Il conviendrait d'analyser l'eau servant au rinçage des bidons dans les fermes suspectes pour confirmer cette seconde hypothèse.

Plus problématique, la présence de YE dans le lait pasteurisé peut être interprétée de plusieurs manières : résistance de certaines souches à la chaleur, défaillance accidentelle du cycle de pasteurisation, recontamination terminale... La négativité de l'épreuve de la phosphatase, systématiquement contrôlée, atteste de la conduite normale de la pasteurisation. Les deux autres causes sont plus difficiles à analyser, et nous ne disposons d'aucun argument en faveur de l'une ou de l'autre actuellement. Notons seulement que dans 2 cas sur 3 , les souches isolées sont de types couramment isolés du lait cru $\left(1-5-\mathrm{Xz}\right.$ et $\left.1-13,7,8,19-\mathrm{Xo} \mathrm{lac}^{+} \mathrm{raf}^{+}\right)$, la troisième étant unique en son genre (1-12-Xz). Des recherches complémentaires sont nécessaires pour évaluer l'importance réelle de ces résultats sur le plan de l'hygiène du lait.

Bien que les épreuves de virulence " in vitro " aient été négatives pour les 112 souches examinées, il serait hasardeux de conclure à leur absence totale de pouvoir pathogène, d'autant plus que nous n'avons pas recherché chez celles-ci la production éventuelle d'entérotoxine. Cette question importante fera l'objet d'un travail ultérieur. 


\section{Remerciements}

Nous adressons nos sincères remerciements au $\operatorname{Pr} \mathrm{H} . \mathrm{H}$. Mollaret qui a confirmé l'identité et effectué le typage de nos souches, et au Pr M. Hasselmann pour l'aide matérielle qu'il nous a apportée.

Nous remercions également tous les techniciens du Laboratoire Interrégional de la Direction de la Consommation et du Larebron qui ont contribué à la réalisation de ce travail.

Nous tenons aussi à exprimer notre gratitude à M. Facq qui nous a beaucoup aidé pendant son séjour au Laboratoire.

\section{Bibliographie}

Aldova (E.) Cerna J., Janeckova (M.) and Pegrimkova (J.) (1975), - Yersinia enterocolitica and its demonstration in the food. Cesk. Hyg., 8, 395-404.

Bercovier (H.), Ursing (J.), Brenner (D. J.), Steigerwalt (A. G.), Fanning (G. R.), CARTer (G. P.) and Mollaret (H. H.) (1980). - Yersinia kristensenii: a new species of Enterobacteriaceae composed of sucrose-negative strains (formerly called atypical Yersinia enterocolitica or Yersinia enterocolitica-like). Curr. Microbiol., 4, 219-224.

Black (R. E.), Jackson (R. J.), Tsai (T.), Medvesky (M.), Shayegani (M.), Feeley (J. C.), MAC LeOD (K. I. E.), and WAKELEe (A. M.) (1978). - Epidemic Yersinia enterocolitica infection due to contaminated chocolate milk. N. Engl. J. Med., $298,76-79$.

CHRISTENSEN (S. G.) (1982). - The prevalence of Yersinia enterocolitica in slaughter animals, water and raw milk in Denmark. In Psychrotrophic Microorganisms in Spoilage and Pathogenicity. Ed. Roberts, T.A., Hobbs, G. Christian, J. H. B. \& Skovgaard, N. London. Academic Press.

De Grace (M.), Laurin (M. F.), Belanger (C.), Rolland (P. E.), Bläis (R.), Breton (J. P.) and MARTINeAU (G.) (1976). - Yersinia enterocolitica gastro-enteritis outbreak-Montreal. Can. Dis. Week. Rep., 2, 41-42.

Delmas (C.) (1982), - Contribution à l'étude des souches de Yersinia enterocolitica isolées du lait. Thèse Doct. 3 cycle Pharm. Strasbourg.

Gemski (P.), Lazere (J. R.) and Casey (T.) (1980). - Plasmid associated with pathogenicity and calcium dependance of Yersinia enterocolitica. Infect. immun., 26 682-685,

Gutman (L. T.), Ottesen (E. A.), Quan (T. J.), Noce (P. S.) and Katz (S. L.) (1973). An interfamilial outbreak of Yersinia enterocolitica enteritis. N. Engl. J. Med., 288, 1372-1377.

Hughes (D.) (1979). - Isolation of Yersinia enterocolitica from milk and a dairy farm in Australia. J. Appl. Bacteriol., 46, 125-130.

Hughes (D.) (1980). - Repeated isolation of Yersinia enterocolitica from pasteurized milk in a holding vat at a dairy factory. J. Appl. Bacteriol., 48, 383-385.

HugHeS (D.) and JENSEN (N.) (1981). - Yersinia enterocolitica in raw goat's milk. Appl. Environ. Microbiol., 41, 309-310.

KaSATIYA (S. S.) (1976). - Yersinia enterocolitica gastroenteritis outbreak-Montreal. Can. Dis. Week. Rep., 2, 73-74.

LAIRD (W. J.) and CaVAnaugr (D. C.) (1980). - Correlation of autoagglutination and virulence of Yersinae. J. Clin. Microbiol., 11, 430-432.

Lauret (O.) (1981). - Recherche de Yersinia enterocolitica dans le lait cru. Résultats d'une enquête et intérêt épidémiologique. Thèse Doct. Vét. Toulouse. 
Megraud (F.), Morales (P.), Beau (M.) et Latrille (J.) (1981). - Yersinia enterocolitica en région bordelaise. Méd. Mal. Infect., 11, 691-694.

Melis (R.) et Mollaret (H. H.) (1981). - Présence de Yersinia enterocolitica dans les eaux. Etat actuel des connaissances. J. Fr. Hydro., 12,175-186.

Mollaret (H. H.) (1976). - Contribution à l'étude épidémiologique des infections à Yersinia enterocolitica. III. Bilan provisoire des connaissances. Méd. Mal. Infect., 6, 442-448.

Portnoy (D. A.), Moseley (S. L.) and Falkow (S.) (1981). - Characterization of plasmids and plasmid-associated determinants of Yersinia enterocolitica pathogenesis. Infect. Immun., 31, 775-782.

Ratnam (S.), Mercer (E.), Picco (B.), Parsons (S.) and Butler (R.) (1982). A nosocomial outbreak of diarheal disease due to Yersinia enterocolitica serotype 0:5, biotype 1. J. Infect. Dis., 145, 242-247.

SARROUY (J.) (1972). - Isolement d'une Yersinia enterocolitica à partir du lait. Méd. Mal. Infect., 2, 67.

Schiemann (D. A.) (1978). - Association of Yersinia enterocolitica with the manufacture of cheese and occurence in pasteurized milk. Appl. Environ. Microbiol., 36, 274-277.

Schiemann (D. A.) and Toma (S.) (1978). - Isolation of Yersinia enterocolitica from raw milk. Appl. Environ. Microbiol., 35, 54-58.

Ursing (J.), Brenner (D. J.), Bercovier (H.), Fanning (G. R.), Steigerwalt (A. G.), Brault (J.) and Mollaret (H. H.) (1980). - Yersinia frederiksenii: a new species of enterobacteriaceae composed of rhamnose-positive strains (formerly called atypical Yersinia enterocolitica or Yersinia enterocolitica-like. Curr. Microbol., 4, 213-217.

Vidon (D. J. M.) and Delmas (C. L.) (1981). - Incidence of Yersinia enterocolitica in raw milk in eastern France. Appl. Environ. Microbiol., 41, 355-359.

Vidon (D. J. M.), LetT (M. C.) and Delmas (C. L.) (1981). - Mercury resistance in Yersinia enterocolitica. Ann. Microbiol. (Inst. Pasteur), 132B, 225-230.

WAuters (G.) (1970). - Contribution à l'étude de Yersinia enterocolitica. Thèse Agreg. Louvain. Vauder éd. 